\title{
Zweite Generation der Immunmodulatoren auch beim Rezidiv hochaktiv
}

\author{
Patienten mit multiplem Myelom haben in den letzten Jahren maßgeb- \\ lich von der Einführung neuer Medikamente wie der Immunmodulatoren \\ vom Typ der IMiDs profitiert. Die nächste Generation dieser Substanzen \\ scheint die Wirksamkeit der Vorgänger noch zu übertreffen.
}

Wie Xavier Leleu, Lille, Frankreich, berichtete, hatte Pomalidomid in Kombination mit niedrig dosiertem Dexamethason bereits in der ersten Phase-II-Studie mit 60 Patienten mit rezidiviertem oder refraktärem Myelom bei $63 \%$ der Patienten $z u$ einer Remission geführt, darunter $5 \%$ komplette und $28 \%$ sehr gute partielle Remissionen. $40 \%$ der Patienten waren gegen Lenalidomid refraktär. Zytogenetische oder molekulare HochrisikoMarker wirkten sich prognostisch nicht negativ aus: Hier lag die Ansprechrate sogar bei $74 \%$. In weiteren Phase-II-Studien, in denen die Patienten bis zu sechs vorangegangene Therapien erhalten hatten, erzielte Pomalidomid immer noch Ansprechraten zwischen 25 und $35 \%$.

In einer von Leleu selbst durchgeführten Studie lag die mediane Zeit bis zur
Progression bei stark vorbehandelten $\mathrm{Pa}$ tienten unter Pomalidomid bei 9,1 Monaten; die Patienten, die ansprachen, hatten davon beim progressionsfreien Überleben mit median 11,3 Monaten einen deutlichen und hochsignifikanten Vorteil gegenüber den Patienten, die unter Pomalidomid lediglich eine Krankheitsstabilisierung erzielt hatten (median 3,8 Monate; Hazard Ratio 0,29; $\mathrm{p}=0$,0005). Wie Leleu ergänzte, sei die progressionsfreie Überlebenszeit unter Pomalidomid auch deutlich länger als unter der jeweils letzten Myelom-Therapie. JosefGulden

„International Myeloma Foundation EHA Journalists' Workshop" am 15.6.2012 in Amsterdam anlässlich des 17th Congress of the European Hematology Association (EHA); Veranstalter: Celgene.

\section{Romiplostim - eine Option für die Zweitlinientherapie}

Über $50 \%$ der Immunthrombozytopenie (ITP)-Patienten lehnen eine Splenektomie als Zweitlinientherapie ab. Für sie sind ThrombopoetinRezeptor-Agonisten eine Alternative

Für die Behandlung der Immunthrombozytopenie (ITP) wurden in den vergangenen Jahren sowohl in den USA als auch in Europa Leitlinien erarbeitet. Die TherapieIndikation orientiere sich primär an der Blutungsneigung, erklärte Axel Matzdorff, Saarbrücken. Steroide werden übereinstimmend für die Erstlinientherapie empfohlen. In der Zweitlinie ist in Deutschland die Splenektomie erste Option, wenn die Blutungen klinisch relevant sind, die ITP schon länger besteht und eine Spontanre- mission unwahrscheinlich ist [Matzdorff A et al. Onkologie. 2010;33 (Suppl 3):2-20]. Aber etwa $80 \%$ der betroffenen Patienten lehnen die Splenektomie ab und bevorzugen eine medikamentöse Therapie. Für sie war die Entwicklung der ThrombopoetinRezeptor-Agonisten (TRAs), wie z.B. Romiplostim $\left(\right.$ Nplate $\left.^{\circ}\right)$ ein großer Fortschritt, auch wenn diese Therapie off-label erfolgt. Eine Splenektomie sollte dennoch erwogen werden, wenn unter TRA-Behandlung innerhalb von zwölf Monaten keine Remission eintrete, so Matzdorff. Günter Springer

19. Münchener Fachpresse-Workshop „Supportive Onkologie und Immunthrombozytopenie", am 12. Juli 2012 in München; unterstützt durch Amgen

\section{G-BA erkennt Zusatznutzen der Immuntherapie an}

Der Gemeinsame Bundesausschuss (G-BA) hat am 2. August 2012 im Rahmen der frühen Nutzenbewertung einen auf fünf Jahre befristeten „Hinweis für einen beträchtlichen Zusatznutzen“ für Ipilimumab (Yervoy ${ }^{\circledR}$ ) beschlossen. Die Entscheidung basiert auf dem statistisch signifikanten Überlebensvorteil gegenüber der zweckmäßigen Vergleichstherapie (Best Supportive Care). In der klinischen Phase-III-Zulassungsstudie konnte gezeigt werden, dass Ipilimumab das Überleben vorbehandelter Patienten mit fortgeschrittenen (nicht-resezierbaren oder metastasierten) Melanomen signifikant verlängert [Hodi FS et al. N Engl J Med. 2010;363(8):711-23. Robert C et al. N Engl J Med. 2011;364(26):2517-26.] Das 1- und 2-Jahres-Überleben konnte fast verdoppelt werden; bei manchen Patienten betrug das Langzeitüberleben mehr als vier Jahre. Die am häufigsten beobachteten Nebenwirkungen resultieren aus der erhöhten oder übermäßigen Immunaktivität. Diese waren allerdings, einschließlich schwerwiegender Ereignisse, gut beherrschbar. Doris Berger

Nach Informationen von Bristol-Myers Squibb

\section{Zulassungsempfehlung für Decitabin ausgesprochen}

Der Ausschuss für Humanarzneimittel Committee für Medical Products for Human Use (CHMP) der Europäischen Arzneimittelbehörde hat die Zulassung für den Einsatz von Decitabin bei der akuten myeloischen Leukämie (AML) bei über 65-jährigen Patienten erteilt, für die die Standardtherapie nicht infrage kommt. Die Bewertung beruht auf den Ergebnissen der im Juni veröffentlichten Daco-016-Studie [Kantarjian HM et al. J Clin Oncol. 2012;30(21):2670-7]. In dieser Untersuchung hatten die Patienten besser auf eine Decitabin-Therapie als auf Cytarabin oder Best Supportive Care angesprochen. Und dies bei vergleichbarer Verträglichkeit. Kantarjian und Kollegen sehen zudem Hinweise auf einen leichten Überlebensvorteil durch die Decitabin-Behandlung.

Nach Informationen von Janssen-Cilag 